OPEN ACCESS

Edited by:

Carla Cannizzaro,

University of Palermo, Italy

Reviewed by:

Olga M. Bazanova,

State Scientific Research Institute

of Physiology and Basic Medicine,

Russia

Walter Adriani,

Istituto Superiore di Sanità (ISS), Italy

*Correspondence: Aleksandra M. Herman aleksandra.herman@rhul.ac.uk;

a.herman@sussex.ac.uk

Specialty section:

This article was submitted to Individual and Social Behaviors, a section of the journa

Frontiers in Behavioral Neuroscience

Received: 01 October 2019

Accepted: 05 June 2020

Published: 24 June 2020

Citation:

Herman AM, Critchley HD and

Duka T (2020) Trait Impulsivity Associated With Altered Resting-State Functional Connectivity Within the Somatomotor Network. Front. Behav. Neurosci. 14:111 doi: 10.3389/fnbeh.2020.00111

\section{Trait Impulsivity Associated With Altered Resting-State Functional Connectivity Within the Somatomotor Network}

\author{
Aleksandra M. Herman ${ }^{1,2 *}$, Hugo D. Critchley ${ }^{3,4}$ and Theodora Duka ${ }^{2,5}$ \\ ${ }^{1}$ Department of Psychology, Royal Holloway, University of London, Egham, United Kingdom, ${ }^{2}$ Behavioural and Clinical \\ Neuroscience, University of Sussex, Brighton, United Kingdom, ${ }^{3}$ Brighton and Sussex Medical School, Brighton, \\ United Kingdom, ${ }^{4}$ Sackler Centre for Consciousness Science, University of Sussex, Brighton, United Kingdom, ${ }^{5}$ Sussex \\ Addiction Research and Intervention Centre, Brighton, United Kingdom
}

Knowledge of brain mechanisms underlying self-regulation can provide valuable insights into how people regulate their thoughts, behaviors, and emotional states, and what happens when such regulation fails. Self-regulation is supported by coordinated interactions of brain systems. Hence, behavioral dysregulation, and its expression as impulsivity, can be usefully characterized using functional connectivity methodologies applied to resting brain networks. The current study tested whether individual differences in trait impulsivity are reflected in the functional architecture within and between restingstate brain networks. Thirty healthy individuals completed a self-report measure of trait impulsivity and underwent resting-state functional magnetic resonance imaging. Using Probabilistic Independent Components Analysis in FSL MELODIC, we identified across participants 10 networks of regions (resting-state networks) with temporally correlated time courses. We then explored how individual expression of these spatial networks covaried with trait impulsivity. Across participants, we observed that greater self-reported impulsivity was associated with decreased connectivity of the right lateral occipital cortex (peak mm 46/-70/16, FWE 1-p = 0.981) with the somatomotor network. No supratheshold differences were observed in between-network connectivity. Our findings implicate the somatomotor network, and its interaction with sensory cortices, in the control of (self-reported) impulsivity. The observed "decoupling" may compromise effective integration of early perceptual information (from visual and somatosensory cortices) with behavioral control programs, potentially resulting in negative consequences.

Keywords: trait impulsivity, resting state, functional connectivity, Barratt Impulsiveness Scale, somatomotor network

\section{INTRODUCTION}

Self-control allows people to make plans for the future, choose the best option from several alternatives, control impulses, inhibit unwanted thoughts, and regulate behaviors and emotions (Kelley et al., 2015). Past studies typically employed task-related functional magnetic resonance imaging (fMRI) to understand the neural substrates of transient fluctuations in self-control 
in different circumstances or in distinct populations. Although this approach is well-suited to capture momentary changes in brain activity in response to specific (internal or external) stimuli, it is arguably insufficient to capture more tonic aspects of selfcontrol (Kelley et al., 2015). A global whole-brain network approach can provide more comprehensive insight into neural substrates supporting individual differences in the capacity for self-control over longer timescales. Moreover, measurement of functional connectivity (FC) across "resting-state" (RS) networks has proven value as a tool for characterizing mechanisms underlying neurocognitive processes and psychiatric disorders, while overcoming technical and inferential limitations of taskrelated fMRI (De Luca et al., 2006; van den Heuvel and Hulshoff Pol, 2010; Cole et al., 2014; Dipasquale et al., 2015).

Specific studies using FC at rest have tested for differences in the interaction between brain regions that account for impulsivity and, more generally, the executive function and dysfunction, in children (Inuggi et al., 2014) and in young adults (Davis et al., 2013; Reineberg et al., 2015). In typically developing children (8-12 years old) parental ratings of trait impulsivity are related to lower RS brain connectivity within the default mode network (DMN), specifically between posterior cingulate cortex and right angular gyrus (Inuggi et al., 2014). The DMN is considered a "task-negative" network, where activity is strongest when an individual is not engaged in an external task (e.g., at rest). Correspondingly, DMN activity is typically anti-correlated to other "task-related" resting-state networks (RSN) (Uddin et al., 2009). In highly impulsive children, the canonical antiphasic relation between the DMN and action-related networks is much reduced, indicating that trait impulsivity is linked to a reduced functional segregation of task-negative and taskpositive networks (i.e., the natural degree of anti-correlation between these networks is reduced). By extension, impulsivity may putatively arise in the context of functional interference between brain systems directing internal and external attention (Inuggi et al., 2014).

In adults, self-report questionnaires are used to assess trait impulsivity, measuring one's tendencies to show premature, unplanned and short-sighted actions and decisions in daily life (Herman et al., 2018). Applying graph-theory approaches to functional brain architecture at rest in adults revealed an association between trait impulsivity and increased segregation between cortical and sub-cortical regions (i.e., increased "modularity") (Davis et al., 2013). This is coherent with findings in young adults for whom core aspects of executive function (quantified using three behavioral tasks) were positively associated with connectivity between the frontal pole and an "attentional" RSN, and also between the cerebellum and a right frontoparietal RSN. This suggests that individuals with better executive functioning manifest more expanded yet more integrated RSN relative to individuals with worse executive functioning (Reineberg et al., 2015). However, there are also contrasting findings: Individuals with increased motor impulsivity (i.e., poorer inhibitory capacity on the go/no-go task) and higher trait impulsivity (Barratt Impulsiveness Scale), reportedly show greater RS FC between the basal ganglia and thalamus, motor cortex, temporal lobe and prefrontal cortex (Korponay et al., 2017). This suggests that increased connectivity between motor-brain regions may predispose to disinhibited actions.

The comparison between these earlier studies to disentangle the observed differences, however, is hindered by the different approaches to functional connectivity (e.g., focus on a single, pre-defined RSN, or the use of seed-based methods, instead of a whole-brain model-free approach) and diverse measures of disinhibited behaviors used (various behavioral tasks or trait measures). Their focus on general executive functioning instead of trait impulsivity or a specific behavioral impulsivity task is also a limitation. The present study set out to cover these gaps by testing for predicted associations between trait impulsivity and the strength of FC within as well as between restingstate networks.

An understanding of the brain mechanisms underlying selfregulation can provide valuable insights into how people regulate and control their thoughts, behaviors, and emotional states and can illuminate what happens on those occasions when this regulation fails (Kelley et al., 2015). The present study tested whether individual differences in trait impulsivity are reflected in within-and between-resting-state network architecture using a FC approach. Based on previous findings, we predicted that internal architecture of the default mode (Inuggi et al., 2014), frontoparietal, and attentional networks (Reineberg et al., 2015) would be linked to the expression impulsivity across individuals and that between-network connectivity pattern of task-negative $(\mathrm{DMN})$ and task-positive networks (Inuggi et al., 2014) might also be modulated by the magnitude of trait impulsivity.

\section{MATERIALS AND METHODS}

\section{Participants}

Thirty volunteers (nine males) were recruited from staff and students of the University of Sussex. Participants were required to be between 18 and 40 years old and right-handed. Exclusion criteria included history of any psychological or neurological disorders, head injury, current treatment for any psychological or physical condition (including use of inhalers; excluding the contraceptive pill), pregnancy or breastfeeding, clinically significant impairment of vision, use of psychoactive substances $48 \mathrm{~h}$ before testing, and any MRI contradictions (claustrophobia, having any metal implants, teeth braces or bridges, or cardiac pacemakers).

All participants provided written informed consent. The study was conducted according to the Declaration of Helsinki. All procedures were approved by the Brighton and Sussex Medical School Research Governance and Ethics Committee.

\section{Questionnaires}

Participants completed the Barratt Impulsiveness Scale (BIS; Patton et al., 1995), a 30-item questionnaire with three distinct impulsivity facets: attentional (eight items; a lack of focus on the ongoing task), motor (11 items; acting without thinking), and non-planning impulsivity (11 items; orientation to the present rather than to the future). 


\section{MRI Experiment Design}

In the MRI scanner, first, a structural scan was obtained followed by a 7 -min resting-state scan (165 volumes) during which participants were instructed to rest with their eyes open focusing on a fixation cross in the center of the screen with the instruction to try not to think of anything and not to fall asleep. All participants were tested between $2 \mathrm{pm}$ and $6 \mathrm{pm}$ to control for possible time of day effects on an attentional level.

\section{MRI Acquisition}

MRI was performed on a 1.5-Tesla MAGNETOM Avanto scanner (Siemens AG, Munich, Germany) with upgraded gradients and a 32-channel headcoil. Structural volumes were obtained using a high-resolution three-dimensional magnetization prepared rapid acquisition gradient echo sequence. Functional data sets used $\mathrm{T} 2{ }^{*}$-weighted echo planar imaging sensitive to blood oxygenation-level-dependent signal (repetition time $=2.52 \mathrm{~s}$, echo time $=43 \mathrm{~ms}$, flip angle $=90^{\circ}$, 34 slices, $3-\mathrm{mm}$ slice thickness, field of view $=192 \mathrm{~mm}$, voxel size $=3 \times 3 \times 3 \mathrm{~mm}$ ). Slices were angled $-30^{\circ}$ in the anteroposterior axis to reduce the signal loss in orbitofrontal regions (Deichmann et al., 2003; Weiskopf et al., 2006).

\section{fMRI Data Pre-processing}

Imaging analysis was performed using FEAT (FMRI Expert Analysis Tool) version 6.00, a part of FMRIB Software Library (FSLv6.0, Jenkinson et al., 2012). Pre-processing steps included (1) skull stripping of structural images with Brain Extraction Tool (BET), (2) removal of the first four functional volumes to allow for signal equilibration, (3) head movement correction by volume-realignment to the middle volume using MCFLIRT, (4) global 4D mean intensity normalization, (5) spatial smoothing (6mm full-width half-maximum), and (6) noise signals removal, (7) temporal high-pass filtering (100 s cut-off).

FMRI datasets were co-registered to the participant's structural image using affine boundary-based registration as implemented in FSL FLIRT (Jenkinson and Smith, 2001; Jenkinson et al., 2002) and subsequently transformed them to MNI152 standard space with $2 \mathrm{~mm}$ isotropic resolution using non-linear registration through FSL FNIRT (Andersson et al., 2010). Noise signals were identified individually and removed using ICA-AROMA toolbox (Pruim et al., 2015). ICA-AROMA incorporates probabilistic Independent Component Analysis (ICA) on the partly pre-processed single-subject fMRI data (following spatial smoothing and normalization but before high-pass filtering), identifies independent components (ICs) representing motion artifacts and removes them from the fMRI time-series using linear regression.

Since there was a broad age range within our population (1837 years) and a larger number of females than males participated in the study, in all reported analyses, gender and mean-centered age were added as covariates of no interest.

\section{Independent Components Analysis}

The RS data analysis pipeline is summarized in Figure 1. To decompose the RS data into various independent spatiotemporal components, Probabilistic Independent Components Analysis
(PICA) was performed on the pre-processed functional scans using Melodic version 3.14 (Beckmann and Smith, 2004). A dimensionality estimation using the Laplace approximation to the Bayesian evidence of the model order (Beckmann and Smith, 2004) produced 11 spatiotemporal components. Following an approach described in Reineberg et al. (2015), we statistically compared the spatial map of each independent component (IC) to a set of seven reference RS networks from a previous largescale RS analysis (Yeo et al., 2011). We used FSL's "fslcc" tool to calculate Pearson's $r$ for each pairwise relationship and kept only those ICs that yielded a significant spatial correlation (Pearson's $r>0.3$ ) with one of the reference networks. This procedure identified and helped label 10 target ICs (see Table 1 for details). Upon visual inspection, the remaining 1 IC was considered noise and was not subjected to further analysis.

\section{Dual regression}

For the between-subject analysis, we carried out dual regression, a technique that back-reconstructs each un-thresholded grouplevel component map at the individual subject level, generating participant-specific spatial maps and time courses (Beckmann et al., 2009; Filippini et al., 2009). The dual regression consists of (1) a spatial regression of the group-average set of ICs that produces a set of participant-specific time series, one per group-level component, and (2) a temporal regression of those participant specific time series, resulting in a set of participant-specific spatial maps, one per group-level component (see Figure 1). Participant-specific components are whole brain images. For some individuals, the given IC might be very similar to the group level IC while others might show variations of the group level IC (i.e., have an expanded/constrained network or high/low connectivity of a particular region). Statistical analyses (discussed below) are performed on these whole brain participant-specific ICs to determine areas that covary with trait impulsivity measure, that is BIS total score.

\section{Within-network connectivity}

To quantify the within-network variation in functional connectivity (FC), depending on BIS total score and participantspecific ICs, we carried out voxel-wise regression to assess statistically significant differences in FC in relation to trait impulsivity score. The analysis was conducted using Randomize, FSL's non-parametric permutation testing tool (Winkler et al., 2014), with 5000 permutations and threshold-free cluster enhancement (TFCE) with an alpha level of 0.05 to correct for multiple comparisons. The permutation testing procedure was run for each set of participant-specific ICs (one for each group-level ICs of interest); thus, the resulting statistical images reveal how variation in RS FC (functional connectivity estimates) predict differences in trait impulsivity (see Figure 1). For example, the permutation testing procedure could reveal that individuals with expanded one of the ICs (i.e., expanded to areas outside the areas included in the group-level IC) report greater impulsivity. Following studies using similar procedures (Uddin et al., 2013; Nomi and Uddin, 2015; Reineberg et al., 2015; de Bézenac et al., 2017; Herman et al., 2019), further correction for multiple component testing was not applied. 


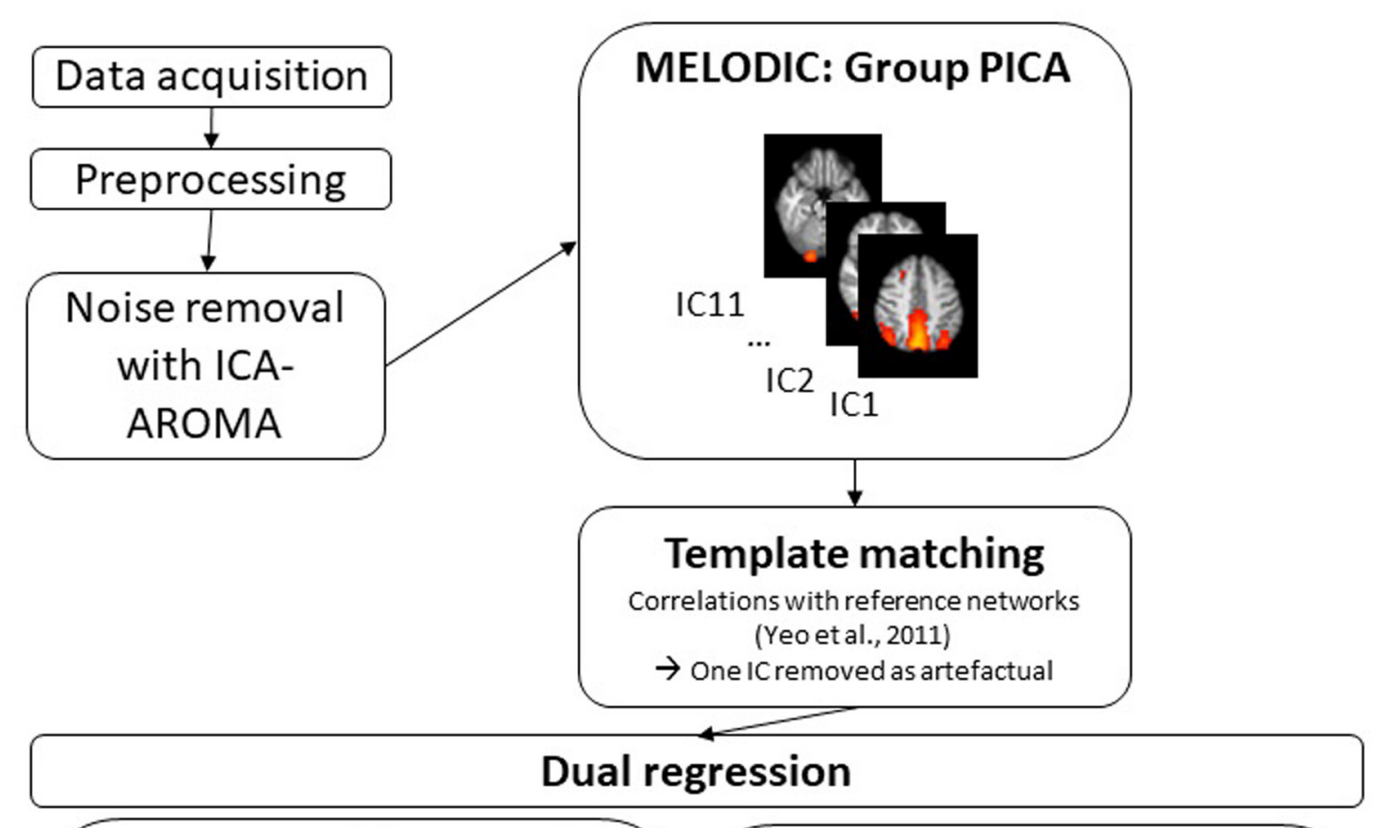

\section{Stage 1: Spatial Regression \\ Stage 2: Temporal Regression}

Participants' $\mathrm{MRI}$ data is regressed against all Group-level IC maps to generate participant-specific timecourses.
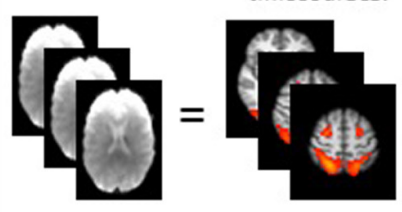

Participants rs-fMRI

data, $\mathrm{N}=30$

Group IC spatial maps,

$I C 1, I C 2, \ldots$. IC10
$\mathrm{X}$

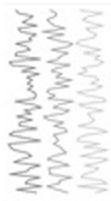

Participantspecific

timecourses, $\mathrm{IC} 1, \mathrm{IC} 2, \ldots . \mathrm{IC} 10$

Participants' fMRI data is regressed against all timecourses derived from stage 1 of dual regression to generate participant-specific spatial map for each IC.

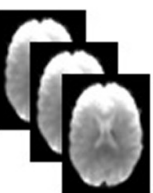

Participants' rs-fMRI

data, $N=30$

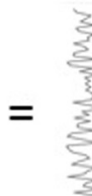

Participantspecific timecourses,

IC1, IC $2, \ldots$. IC10 $\mathrm{x}$

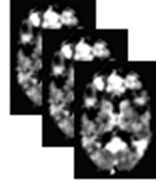

Participantspecific spatial maps IC1, IC2, ... IC10

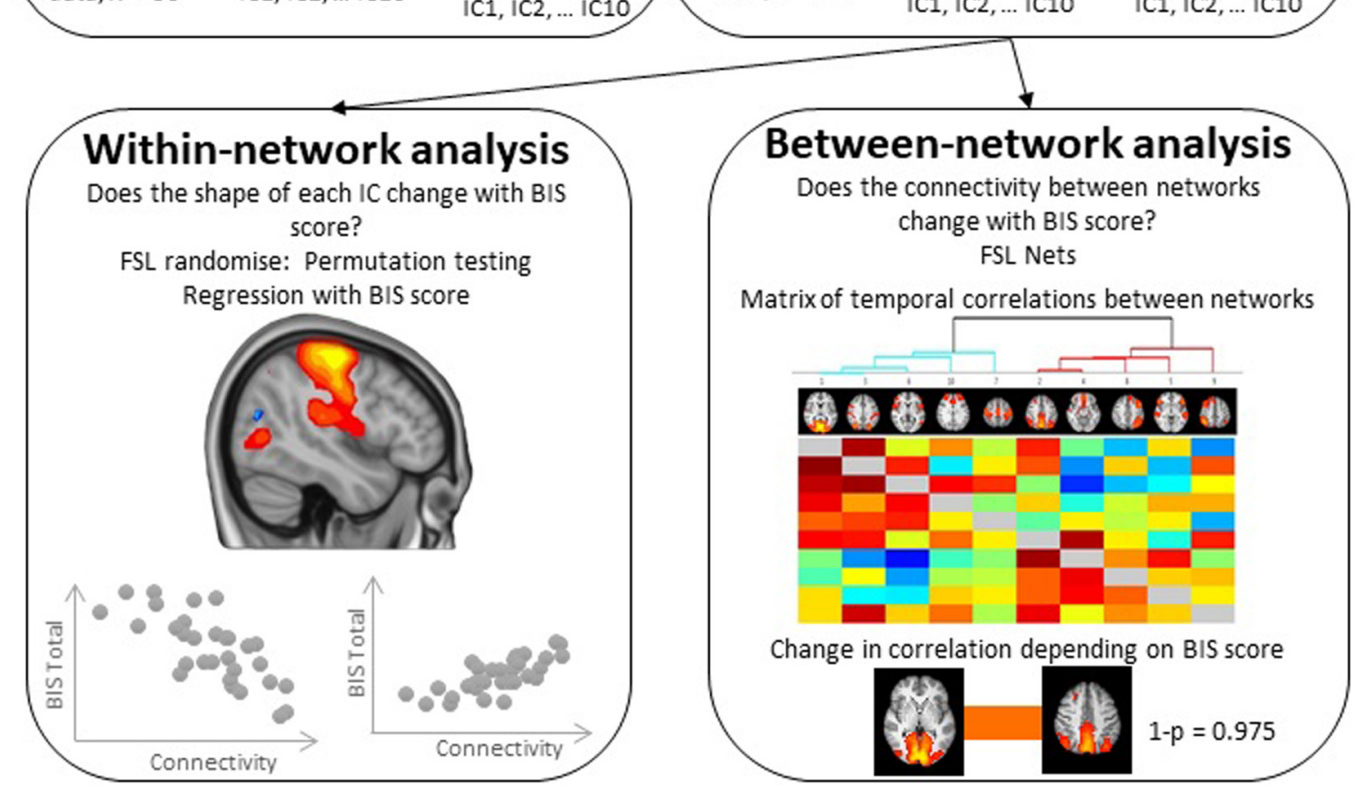

FIGURE 1 | Illustration of the steps followed during resting-state functional $\mathrm{fMRI}$ data analysis. For more details of this analysis steps see Methods section. rs-fMRI, Resting state functional magnetic resonance imaging; PICA, Probabilistic Independent Component Analysis; IC, Independent Component; BIS, Barratt Impulsiveness Scale. 
TABLE 1 | Identified Independent Components (IC Number) and their characteristics.

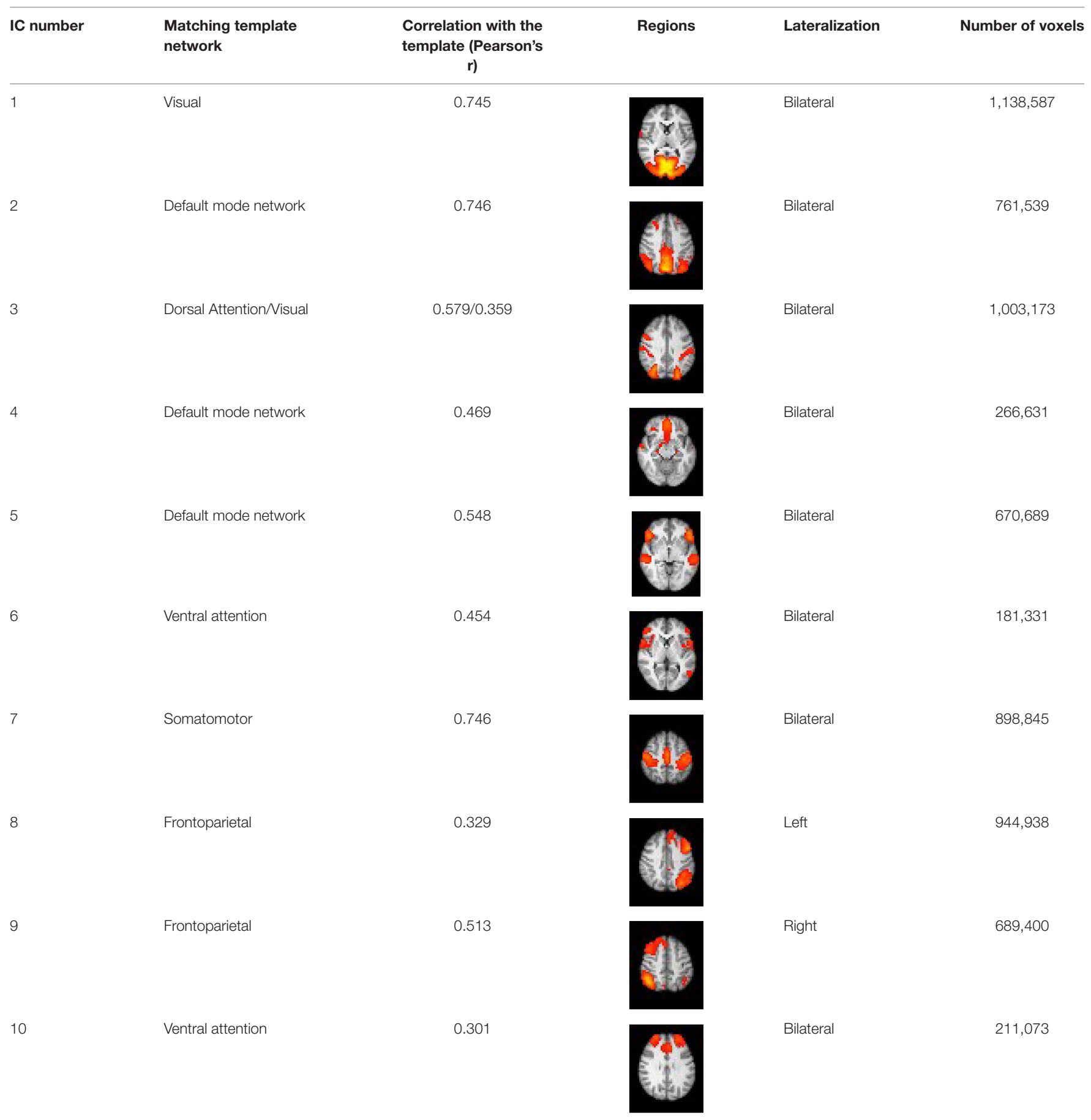

Images are presented in the radiological convention (left side of the brain is presented on the right side of the image). DMN, Default Mode Network.

\section{Between-network connectivity}

FSLNets. To examine the relationship between trait impulsivity and between-network FC, we employed the FSLNets package ${ }^{1}$ implemented in Matlab v2015b (The MathWorks, 2015). This analysis involved correlation of the participants' time courses

${ }^{1}$ http://fsl.fmrib.ox.ac.uk/fsl/fslwiki/FSLNets from the dual regression analysis and subjected them to betweennetwork comparisons to determine how they are correlated with each other (Smith et al., 2013). We then calculated full and partial correlations between all pairs of ICs. Partial correlations are computed as correlations between two ICs while controlling for the effect of all other ICs and are thought to reflect more direct connections (Smith et al., 2011). Finally, BIS total score was 


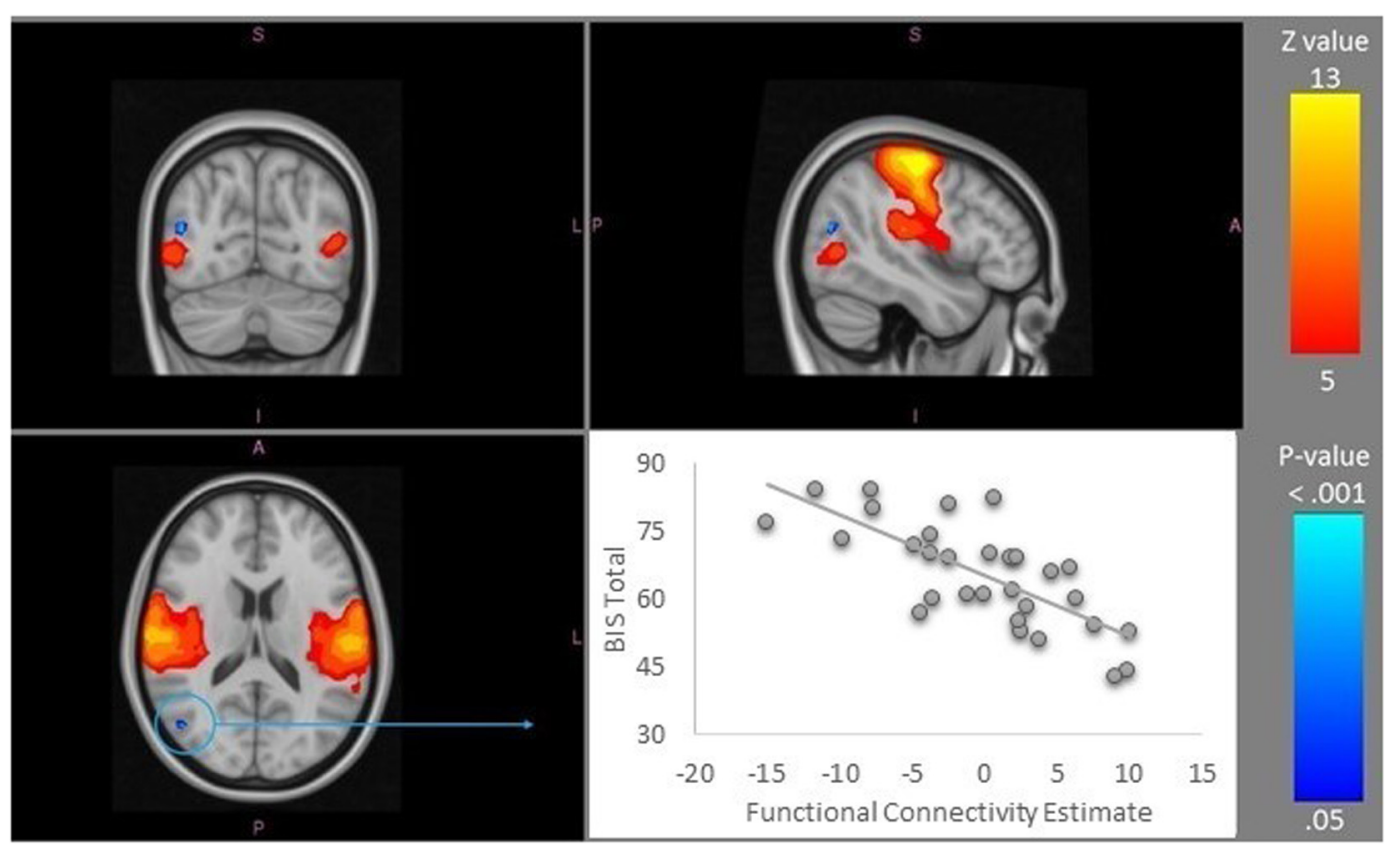

FIGURE 2 | Somatomotor resting-state network (IC 7), depicted in warm colors, was identified as the only network showing the significant differences across the BIS total score spectrum. The area that showed reduced resting state functional connectivity within this network was a region in the lateral occipital cortex (in blue; $X=46, Y=-70, Z=16)$. The visualization of the relationship is shown in the scatterplot in the bottom right corner. Images are presented in the radiological convention. A-anterior, I-inferior, L-left, P-posterior, R-right, S-superior. IC, Independent Component.

used as a regressor in the regression analysis in FSL randomize with 5000 permutations to assess differences in between-network connectivity across BIS spectrum. Results were FWE corrected for multiple comparisons.

\section{RESULTS}

\section{Participants}

No participant was removed because of extensive motion in the scanner. The final sample $(N=30,9$ males) was aged between 18 and 37 years old $(M=23.40, S D=5.01)$. The average BIS Total score was $65.30 \pm 11.39$.

\section{Within-Network Connectivity}

Greater self-reported impulsivity (BIS score) was associated with lower functional connectivity of the right lateral occipital cortex with IC7, a network that correlated significantly with Somatomotor template network (peak mm 46/-70/16, FWE 1$p=0.981)$ (Figure 2).

\section{Between-Network Connectivity}

Network analysis using FSLNets revealed a modular structure of functional networks, which could be segregated into clusters: Cluster 1 comprised of Visual, Somatomotor as well as Ventral and Dorsal Attention Networks (Figure 3, blue cluster), while Cluster 2 comprised of Frontoparietal and Default-Mode Networks (Figure 3, red cluster).
Using BIS as a predictor, no significant between-network differences in connectivity were found.

\section{DISCUSSION}

This study investigated whether aspects of intrinsic functional architecture and between-network connectivity pattern is associated with individual differences in trait impulsivity in a normative (university) population. We showed that individual differences in trait impulsivity, assessed with BIS Total Score, are associated with altered aspects of the functional architecture of the Somatomotor RS network. Specifically, higher trait impulsivity was linked to decreased coupling between the lateral occipital cortex and the Somatomotor Network. Surprisingly, we did not find any significant differences in the network functional architecture of default mode or frontoparietal networks associated with impulsivity, as has been reported previously (Inuggi et al., 2014; Reineberg et al., 2015). However, it is important to note that such previous research used different measures of impulsivity. Therefore, those inconsistent findings might merely reflect a heterogeneous nature of impulsivity and its underlying neural mechanisms (Caswell et al., 2015; Herman et al., 2018).

The finding of disrupted FC within the Somatomotor RS network in relation to trait impulsivity level corroborates previous studies. The graph theory approach, has been used to test the relationship between impulsivity (as reflected in BIS score) and the functional segregation (i.e., modularity) 


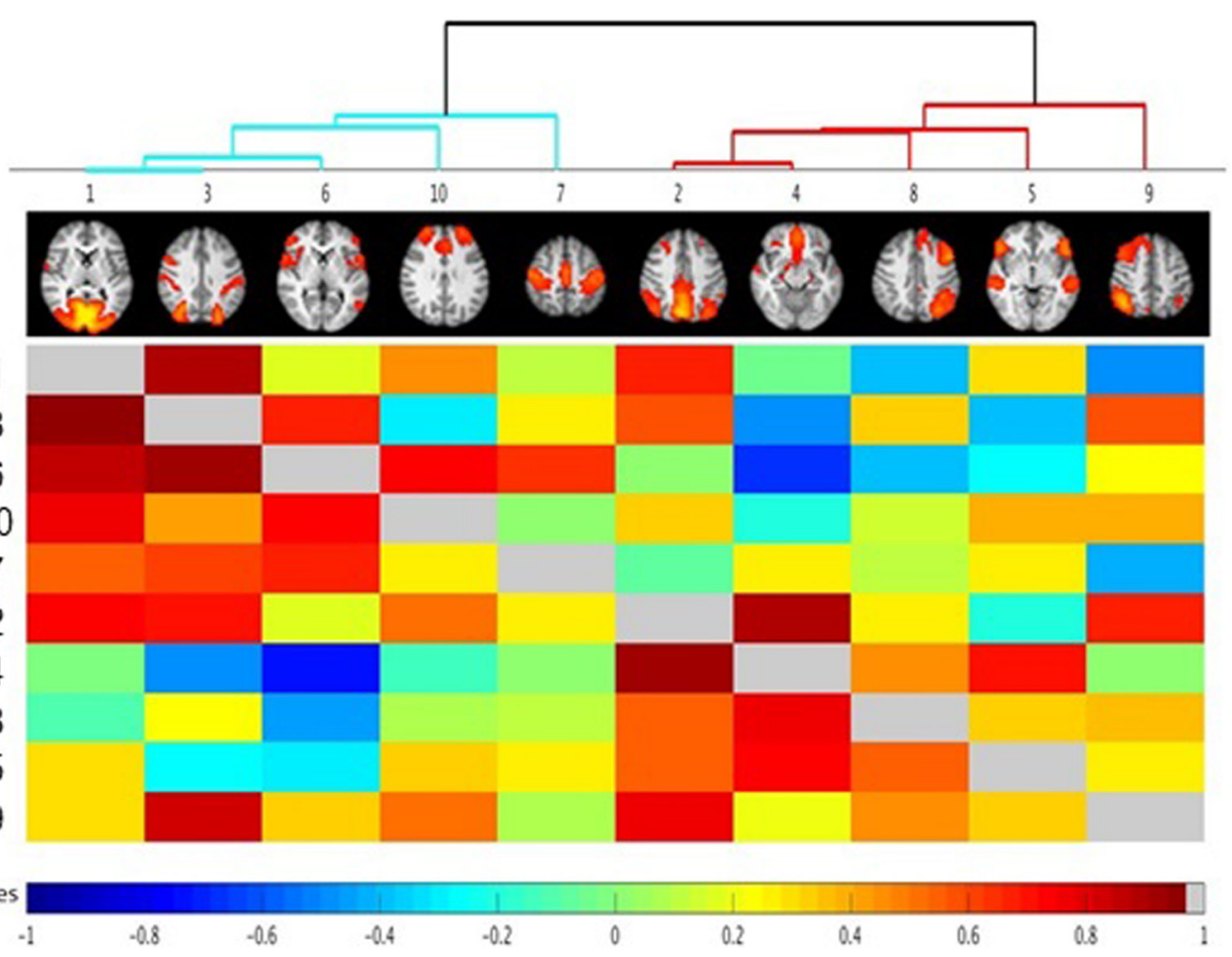

FIGURE 3 | FSLNets results of between network correlations ( $N=30)$. Each independent component (IC) is denoted by one column and a corresponding raw. The colored matrix displays the correlations of the time series between networks pairs. Dark red squares indicate highly positive correlations, light green indicates a near-0 correlation, and dark blue represents highly negative correlation as denoted on the scale at the bottom of the figure. Full correlations between networks pairs are shown below the diagonal line (in gray) with partial correlations shown above the diagonal line (for detailed description of full and partial correlation please see main text). Groups of highly correlated ICs were clustered together according to a hierarchical clustering algorithm (visualized at the top of the matrix as a clustering tree). Please note that the color cut-off for hierarchical tree is arbitrary - just for visualization purposes. Numbers indicate specific independent components as described in Table 1. The ICs have been reordered, according to a hierarchical clustering algorithm. Small images at the top of each column summarize each IC's spatial map, with the right side of the images representing the left side of the brain. As described in the main text, the between-network connectivity was not modulated by trait impulsivity score.

of whole-brain resting state architecture (Davis et al., 2013). Overall, this reveals a shift in the functional connectivity between visual, sensorimotor, cortical, and subcortical structures across the impulsivity range; specifically pointing to increased functional modularity between cortical and sub-cortical regions as a function of impulsivity score.

The lateral occipital cortex supports both visual perception and multisensory integration (Grill-Spector et al., 2001; Beauchamp, 2005). Interestingly, it is recognized that visual cortices contribute to impulsivity (Davis et al., 2013) and disorders commonly associated with impulsivity, including as Attention Deficit-Hyperactivity Disorder (ADHD; Castellanos and Proal, 2013). The sensorimotor network consists of both motor cortices, known to play a critical role in response inhibition (Li et al., 2006; Duque et al., 2012; Rae et al., 2014), and somatosensory areas, which are vital for sensory integration. These regions show altered activity in inhibitory control in diseased states such as post-traumatic stress disorder (Falconer et al., 2008; van Rooij et al., 2014) or under pharmacological interventions with LSD (Schmidt et al., 2017). Here, the "decoupling" may itself reflect a deficit in effective integration of perceptual information (visual and somatosensory cortices) with somatomotor outputs (motor cortex) associated with behavioral control, ultimately resulting in negative consequences, from poor planning for the future to excessive substance use (Dickman, 1990; Herman and Duka, 2019).

\section{Limitations}

Some limitations merit comment. Our study was conducted on a moderately sized sample of students and employees of the university. The average BIS total score in our sample is $65.30 \pm 11.39$; which is consistent with other reports in the literature of university sample [e.g., $65.67 \pm 9.92$ in males and $64.58 \pm 10.36$ in females according to Caswell et al. (2015) and $63.82 \pm 10.17$ according to Patton et al. (1995)] and normative community populations $[59.18 \pm 9.54$ according to Reise et al. (2013) or $62.3 \pm 10.3$ according to Stanford et al. (2009)]. However, our sample consists of relatively highfunctioning young adults, who may have developed many mechanisms to cope with elevated impulsivity levels in daily life, which might have an effect on aspects of functional connectivity. It is also important to mention that the majority 
of the sample consisted of females, some of which were using hormonal contraception, which can affect functional connectivity (Hausmann, 2005). Therefore, future research should replicate our findings in larger-scale studies with general population, including a range of individuals with various backgrounds and educational levels. Finally, we did not find any suprathreshold differences in between-network connectivity that could be related to elevated impulsivity levels. Possibly, this is because our sample consisted of highly functioning young adults, all from the university population, and differences in between-network connectivity may only reveal themselves in pathologically impulsive individuals.

\section{CONCLUSION}

In the brain, aspects of the functional architecture of the Somatomotor Network were associated with individual differences in trait impulsivity (BIS Total score). Specifically, more impulsive individuals showed decreased connectivity between the lateral occipital cortex and the Somatomotor Network. Since perception informs action and vice versa (CreemRegehr and Kunz, 2010), proper integration of sensory inputs is crucial for adaptive behavioral responses. Therefore, the observed decreased connectivity between the visual and somatosensory cortices and motor cortex, may reflect itself in less effective integration of perceptual information and behavioral control and, thus, in negative consequences. However, in this normative sample, the between-network architecture was not related to trait impulsivity level. This evidence supports the use of RS FCapproaches to identify biomarkers for impulse-control problems.

\section{REFERENCES}

Andersson, J. L. R., Jenkinson, M., and Smith, S. (2010). Non-Linear Registration, Aka Spatial Normalisation. Oxford: Oxford Press.

Beauchamp, M. S. (2005). See me, hear me, touch me: multisensory integration in lateral occipital-temporal cortex. Curr. Opin. Neurobiol. 15, 145-153. doi: 10.1016/j.conb.2005.03.011

Beckmann, C. F., Mackay, C., Filippini, N., and Smith, S. (2009). Group comparison of resting-state FMRI data using multi-subject ICA and dual regression. Neuroimage 47:S148. doi: 10.1016/S1053-8119(09)71511-3

Beckmann, C. F., and Smith, S. M. (2004). Probabilistic independent component analysis for functional magnetic resonance imaging. IEEE Trans. Med. Imaging 23, 137-152. doi: 10.1109/TMI.2003.822821

Castellanos, X. F., and Proal, E. (2013). Large-scale brain systems in ADHD: beyond the prefrontal- striatal model. Trends Cogn. Sci. 16, 17-26. doi: 10.1016/ j.tics.2011.11.007

Caswell, A. J., Bond, R., Duka, T., and Morgan, M. J. (2015). Further evidence of the heterogeneous nature of impulsivity. Pers. Individ. Diff. 76, 68-74. doi: 10.1016/j.paid.2014.11.059

Cole, M. W., Repovš, G., and Anticevic, A. (2014). The frontoparietal control system: a central role in mental health. Neuroscientist 20, 652-664. doi: 10.1177/ 1073858414525995

Creem-Regehr, S. H., and Kunz, B. R. (2010). Perception and action. Wiley Interdiscip. Rev. Cogn. Sci. 1, 800-810. doi: 10.1002/wcs.82

Davis, F. C., Knodt, A. R., Sporns, O., Lahey, B. B., Zald, D. H., Brigidi, B. D., et al. (2013). Impulsivity and the modular organization of restingstate neural networks. Cereb. Cortex 23, 1444-1452. doi: 10.1093/cercor/ bhs126

\section{DATA AVAILABILITY STATEMENT}

The datasets generated for this study are available on request to the corresponding author. All behavioral data is available within the manuscript.

\section{ETHICS STATEMENT}

The studies involving human participants were reviewed and approved by the Brighton and Sussex Medical School Research Governance and Ethics Committee. The patients/participants provided their written informed consent to participate in this study.

\section{AUTHOR CONTRIBUTIONS}

$\mathrm{AH}, \mathrm{HC}$, and TD were responsible for the study concept and design. AH carried out the study and the data analysis and drafted the manuscript. AH and TD interpreted the findings. HC and TD provided critical revisions of the manuscript for important intellectual content. All authors contributed to the article and approved the submitted version.

\section{FUNDING}

The study was supported by the Sussex Neuroscience Ph.D. studentship awarded to AH as well as MRC Grant awarded to HC (Grant No. G2038).

de Bézenac, C. E., Sluming, V., and Corcoran, R. (2017). Agency performance modulates resting-state variation in prefrontal brain regions. Neuropsychologia 111, 16-25. doi: 10.1016/j.neuropsychologia.2017.12.035

De Luca, M., Beckmann, C. F., De Stefano, N., Matthews, P. M., and Smith, S. M. (2006). fMRI resting state networks define distinct modes of long-distance interactions in the human brain. Neuroimage 29, 1359-1367. doi: 10.1016/j. neuroimage.2005.08.035

Deichmann, R., Gottfried, J. A., Hutton, C., and Turner, R. (2003). Optimized EPI for fMRI studies of the orbitofrontal cortex. Neuroimage 19, 430-441. doi: 10.1016/S1053-8119(03)00073-9

Dickman, S. J. (1990). Functional and dysfunctional impulsivity: personality and cognitive correlates. J. Pers. Soc. Psychol. 58, 95-102. doi: 10.1037/0022-3514. 58.1 .95

Dipasquale, O., Griffanti, L., Clerici, M., Nemni, R., Baselli, G., and Baglio, F. (2015). High-dimensional ICA analysis detects within-network functional connectivity damage of default-mode and sensory-motor networks in Alzheimer's disease. Front. Hum. Neurosci. 9:43. doi: 10.3389/fnhum.2015. 00043

Duque, J., Labruna, L., Verset, S., Olivier, E., and Ivry, R. B. (2012). Dissociating the role of prefrontal and premotor cortices in controlling inhibitory mechanisms during motor preparation. J. Neurosci. 32, 806-816. doi: 10.1523/JNEUROSCI. 4299-12.2012

Falconer, E., Bryant, R., Felmingham, K. L., Kemp, A. H., Gordon, E., Peduto, A., et al. (2008). The neural networks of inhibitory control in posttraumatic stress disorder. J. Psychiatry Neurosci. 33, 413-422.

Filippini, N., MacIntosh, B. J., Hough, M. G., Goodwin, G. M., Frisoni, G. B., Smith, S. M., et al. (2009). Distinct patterns of brain activity in young carriers of the APOE-epsilon4 allele. PNAS 106, 7209-7214. doi: 10.1073/pnas.0811879106 
Grill-Spector, K., Kourtzi, Z., and Kanwisher, N. (2001). The lateral occipital complex and its role in object recognition. Vis. Res. 41, 1409-1422. doi: 10.1016/ S0042-6989(01)00073-6

Hausmann, M. (2005). Hemispheric asymmetry in spatial attention across the menstrual cycle. Neuropsychologia 43, 1559-1567. doi: 10.1016/j.neuropsychologia.2005.01.017

Herman, A. M., Critchley, H. D., and Duka, T. (2018). The role of emotions and physiological arousal in modulating impulsive behaviour. Biol. Psychol. 133, 30-43. doi: 10.1016/j.biopsycho.2018.01.014

Herman, A. M., Critchley, H. D., and Duka, T. (2019). Binge drinking is associated with attenuated frontal and parietal activation during successful response inhibition in fearful context. Eur. J. Neurosci. 50, 2297-2310. doi: 10.1111/ejn. 14108

Herman, A. M., and Duka, T. (2019). Facets of impulsivity and alcohol use: what role do emotions play? Neurosci. Biobehav. Rev. 106, 202-216. doi: 10.1016/j. neubiorev.2018.08.011

Inuggi, A., Sanz-Arigita, E., González-Salinas, C., Valero-García, A. V., GarcíaSantos, J. M., and Fuentes, L. J. (2014). Brain functional connectivity changes in children that differ in impulsivity temperamental trait. Front. Behav. Neurosci. 8:156. doi: 10.3389/fnbeh.2014.00156

Jenkinson, M., Bannister, P., Brady, M., and Smith, S. (2002). Improved optimization for the robust and accurate linear registration and motion correction of brain images. Neuroimage 17, 825-841. doi: 10.1016/S10538119(02)91132-8

Jenkinson, M., Beckmann, C. F., Behrens, T. E., Woolrich, M. W., and Smith, S. M. (2012). FSL. Neuroimage 62, 782-790. doi: 10.1016/j.neuroimage.2011.09.015

Jenkinson, M., and Smith, S. (2001). A global optimisation method for robust affine registration of brain images. Med. Image Anal. 5, 143-156. doi: 10.1016/S13618415(01)00036-6

Kelley, W. M., Wagner, D. D., and Heatherton, T. F. (2015). In search of a human self-regulation system. Annu. Rev. Neurosci. 38, 389-411. doi: 10.1146/annurevneuro-071013-014243

Korponay, C., Dentico, D., Kral, T., Ly, M., Kruis, A., Goldman, R., et al. (2017). Neurobiological correlates of impulsivity in healthy adults: lower prefrontal gray matter volume and spontaneous eye-blink rate but greater resting-state functional connectivity in basal ganglia-thalamo-cortical circuitry. Neuroimage 157, 288-296. doi: 10.1016/j.neuroimage.2017.06.015

Li, C. R., Huang, C., Constable, R. T., and Sinha, R. (2006). Imaging response inhibition in a stop-signal task: neural correlates independent of signal monitoring and post-response processing. J. Neurosci. 26, 186-192. doi: 10. 1523/JNEUROSCI.3741-05.2006

Nomi, J. S., and Uddin, L. Q. (2015). Developmental changes in large-scale network connectivity in autism. Neuroimage Clin. 7, 732-741. doi: 10.1016/j.nicl.2015. 02.024

Patton, J. H., Stanford, M. S., and Barratt, E. S. (1995). Factor structure of the Barratt impulsiveness scale. J. Clin. Psychol. 51, 768-774. doi: 10.1002/10974679(199511)51:6<768

Pruim, R. H. R., Mennes, M., van Rooij, D., Llera, A., Buitelaar, J. K., and Beckmann, C. F. (2015). ICA-AROMA: a robust ICA-based strategy for removing motion artifacts from fMRI data. Neuroimage 112, 267-277. doi: 10.1016/j.neuroimage.2015.02.064

Rae, C. L., Hughes, L. E., Weaver, C., Anderson, M. C., and Rowe, J. B. (2014). Selection and stopping in voluntary action: a meta-analysis and combined fMRI study. Neuroimage 86, 381-391. doi: 10.1016/j.neuroimage.2013.10.012

Reineberg, A. E., Andrews-Hanna, J. R., Depue, B. E., Friedman, N. P., and Banich, M. T. (2015). Resting-state networks predict individual differences in common and specific aspects of executive function. Neuroimage 104, 69-78. doi: 10.1016/ j.neuroimage.2014.09.045

Reise, S. P., Moore, T. M., Sabb, F. W., Brown, A. K., and London, E. D. (2013). The barratt impulsiveness scale-11: reassessment of its structure in a community sample. Psychol. Assess. 25, 631-642. doi: 10.1037/a0032161

Schmidt, A., Muller, F., Lenz, C., Dolder, P. C., Schmid, Y., Zanchi, D., et al. (2017). Acute LSD effects on response inhibition neural networks. Psychol. Med. 48, 1464-1473. doi: 10.1017/S0033291717002914

Smith, S. M., Miller, K. L., Salimi-Khorshidi, G., Webster, M., Beckmann, C. F., Nichols, T. E., et al. (2011). Network modelling methods for FMRI. Neuroimage 54, 875-891. doi: 10.1016/j.neuroimage.2010.08.063

Smith, S. M., Vidaurre, D., Beckmann, C. F., Glasser, M. F., Jenkinson, M., Miller, K. L., et al. (2013). Functional connectomics from resting-state fMRI. Trends Cogn. Sci. 17, 666-682. doi: 10.1016/j.tics.2013.09.016

Stanford, M. S., Mathias, C. W., Dougherty, D. M., Lake, S. L., Anderson, N. E., and Patton, J. H. (2009). Fifty years of the barratt impulsiveness scale: an update and review. Pers. Individ. Dif. 47, 385-395. doi: 10.1016/j.paid.2009. 04.008

The MathWorks (2015). MATLAB Release 2015b. Vienna: Springer.

Uddin, L. Q., Kelly, A. M. C., Biswal, B. B., Castellanos, F. X., and Milham, M. P. (2009). Functional connectivity of default mode network components: correlation, anticorrelation, and causality. Hum. Brain Mapp. 30, 625-637. doi: 10.1002/hbm.20531

Uddin, L. Q., Supekar, K., Lynch, C. J., Khouzam, A., Phillips, J., Feinstein, C., et al. (2013). Salience network-based classification and prediction of symptom severity in children with autism. JAMA Psychiatry 70, 869-879. doi: 10.1001/ jamapsychiatry.2013.104

van den Heuvel, M. P., and Hulshoff Pol, H. E. (2010). Exploring the brain network: a review on resting-state fMRI functional connectivity. Eur. Neuropsychopharmacol. 20, 519-534. doi: 10.1016/j.euroneuro.2010.03.008

van Rooij, S. J. H., Rademaker, A. R., Kennis, M., Vink, M., Kahn, R. S., and Geuze, E. (2014). Impaired right inferior frontal gyrus response to contextual cues in male veterans with PTSD during response inhibition. J. Psychiatry Neurosci. 39, 330-338. doi: 10.1503/jpn.130223

Weiskopf, N., Hutton, C., Josephs, O., and Deichmann, R. (2006). Optimal EPI parameters for reduction of susceptibility-induced BOLD sensitivity losses: a whole-brain analysis at $3 \mathrm{~T}$ and $1.5 \mathrm{~T}$. Neuroimage 33, 493-504. doi: 10.1016/j. neuroimage.2006.07.029

Winkler, A. M., Ridgway, G. R., Webster, M. A., Smith, S. M., and Nichols, T. E. (2014). Permutation inference for the general linear model. Neuroimage 92, 381-397. doi: 10.1016/j.neuroimage.2014.01.060

Yeo, B. T. T., Krienen, F. M., Sepulcre, J., Sabuncu, M. R., Lashkari, D., Hollinshead, M., et al. (2011). The organization of the human cerebral cortex estimated by intrinsic functional connectivity. J. Neurophysiol. 106, 1125-1165. doi: 10.1152/ jn.00338.2011

Conflict of Interest: The authors declare that the research was conducted in the absence of any commercial or financial relationships that could be construed as a potential conflict of interest.

Copyright (c) 2020 Herman, Critchley and Duka. This is an open-access article distributed under the terms of the Creative Commons Attribution License (CC BY). The use, distribution or reproduction in other forums is permitted, provided the original author(s) and the copyright owner(s) are credited and that the original publication in this journal is cited, in accordance with accepted academic practice. No use, distribution or reproduction is permitted which does not comply with these terms. 